\title{
Enhancement and Suppression of lonization in Drug Analysis Using HPLC-MS/MS in Support of Therapeutic Drug Monitoring: A Review of Current Knowledge of Its Minimization and Assessment
}

\author{
Rani George, MMedRes, * Alison Haywood, PhD, * + Sohil Khan, PhD, * \\ Mirjana Radovanovic, BSc, $\neq$ Joshua Simmonds, BPharmaceutSc, * and Ross Norris, PhD $\$ \$$
}

\begin{abstract}
High-performance liquid chromatography coupled with tandem mass spectrometry is commonly used for quantitation of analytes in biological matrices, because of the selectivity, sensitivity, and high throughput offered by this technique. However, the presence of both suppression and enhancement of ionization (SEI) by matrix components is an increasingly recognized impediment to accurate results. The existence of SEI indicates that ionization efficiency is a result of the chemical environment seen by both the analyte and internal standard during ion formation. SEI is influenced by the type and the make of ion source used, mobile-phase composition, extent of sample preparation, and the ability to chromatographically separate other compounds that may influence ionization of the analyte and/or internal standard. A comprehensive review of the phenomenon of SEI in high-performance liquid chromatography coupled with tandem mass spectrometry was conducted, and a summary of salient papers relating to therapeutic agents in biological matrices is presented. Suggestions for approaches to minimize, normalize, or assess SEI and its deleterious effect on accuracy and sensitivity, and hence the validity of quantitative results, are provided. Consideration is also given to a strategy to test for SEI, including the number of samples from different sources that are required to adequately test for SEI.
\end{abstract}

Received for publication May 4, 2016; accepted July 5, 2017.

From the *School of Pharmacy, Menzies Health Institute Queensland, Griffith University, Gold Coast; †Master Research, The University of Queensland, South Brisbane, Queensland; †SydPath, St. Vincent’s Hospital; §Clinical School, University of New South Wales, Sydney, New South Wales; and TSchool of Pharmacy, Griffith University, Nathan, Queensland, Australia. The authors declare no conflict of interest.

Supplemental digital content is available for this article. Direct URL citations appear in the printed text and are provided in the HTML and PDF versions of this article on the journal's Web site (www.drugmonitoring.com).

Correspondence: Ross Norris, PhD, Clinical Pharmacology and Toxicology, SydPath, St. Vincent's Hospital, 390 Victoria St, Darlinghurst, New South Wales 2010, Australia (e-mail: Ross.Norris@svha.org.au).

Copyright (C 2017 The Author(s). Published by Wolters Kluwer Health, Inc. on behalf of the International Association of Therapeutic Drug Monitoring and Clinical Toxicology. This is an open-access article distributed under the terms of the Creative Commons Attribution-Non CommercialNo Derivatives License 4.0 (CCBY-NC-ND), where it is permissible to download and share the work provided it is properly cited. The work cannot be changed in any way or used commercially without permission from the journal.
Key Words: ionization suppression, ionization enhancement, HPLCMS/MS, tandem mass spectrometry, ionization, stable isotopes

(Ther Drug Monit 2018;40:1-8)

\section{INTRODUCTION}

Currently, high-performance liquid chromatography coupled with tandem mass spectrometry (HPLC-MS/MS) is one of the most versatile analytical tools available, as evidenced by the rapid and diverse growth in applications ${ }^{1}$ in the analysis of drugs and metabolites from biological fluids, trace analysis in complex mixtures, therapeutic drug monitoring, pharmacokinetic and clinical studies, metabolomics, and toxicology, that is, wherever the quantitation of small molecules is required. However, the advantages of the excellent selectivity, sensitivity, and high throughput inherent to HPLC-MS/MS have been challenged by reports of inaccuracy and reduced sensitivity because of matrix-related ion suppression. ${ }^{1,2}$ Unlike fluorometric and ultraviolet detection, HPLCMS/MS systems require ionization of the analyte before it can be detected. That is, apart from removing the solvent, the MS/ MS must first convert the analyte molecules to ions, to allow entry into the MS/MS. Suppression of ionization in the ion source, relative to that occurring in the calibrators, is commonly known as ion suppression and results in a signal that is not proportional to that of the calibrators, causing errors in quantitation. "Ion enhancement" has also been reported, resulting in inaccurate quantitation. It has been correctly suggested that the terms "ionization suppression" and "ionization enhancement" should be used because they arise from matrixdependent changes in the percentage of ions generated from the analyte in the ion source (ion efficiency). ${ }^{3}$ The potential errors caused by this phenomenon are so significant that suppression of ionization has been described as the "Achilles Heel" of HPLC-MS/MS ${ }^{4}$ for quantitation of small molecules.

HPLC-MS/MS instrumentation consists of a traditional HPLC instrument coupled to a tandem mass spectrometer, although many nowadays often use ultra-high-performance liquid chromatography (UHPLC), which gives improved chromatographic separation using higher pressures than traditional HPLC but is otherwise the same. The tandem mass spectrometer consists of an ion source where the HPLC eluent is introduced, solvent evaporated, and ions formed. The ions 
are then introduced to the tandem mass spectrometer through the orifice. As suggested by the name, the tandem mass spectrometer is the adjoining of 2 traditional mass spectrometers; the first mass spectrometer is selected for the molecular ion and the second is selected for the product ion (s), after passing the molecular ions through a collision cell to produce characteristic fragments.

Suppression and enhancement of ionization (SEI) may cause erroneous analytical results if the ionization efficiency for the samples being analyzed differs from that of the calibrators against which they are quantitated. An additional opportunity for error is created by the use of the traditional types of internal standards (ie, analog/s of the drug/s being assayed), which do not usually coelute because the internal standard under these circumstances provides a second opportunity for SEI to impact the assay. Despite the unrivaled potential offered by HPLC-MS/MS, operation in single-ion monitoring or selected reaction monitoring modes is unable to detect the matrix components other than those for which the mass-to-charge $(\mathrm{m} / \mathrm{z})$ ratio is known and specifically monitored. These matrix components may therefore cause SEI without being detected themselves and undermine the fundamental assumption that signal response for the analyte in the unknown sample is the same as that in the calibrator, at the same concentration.

Guidelines for method development and validation, issued by regulatory authorities including the Food and Drug Administration (FDA), ${ }^{5}$ emphasize the need for the assessment of matrix effects during development and validation of HPLC-MS/MS methods "to ensure that precision, selectivity, and sensitivity will not be compromised." The European Medicines Agency Guideline on bioanalytical method validation $^{6}$ also details a recommended approach to evaluation of matrix effects (including suppression of ionization). Several approaches have been adopted to minimize or normalize for suppression of ionization, which require extensive understanding of a number of factors, including the type of ion source, solvents used, the analyte, and the sample matrices being analyzed. ${ }^{7}$

This article provides a review of the published literature to explore the phenomenon of SEI in HPLC-MS/MS, its minimization, and assessment. Suggestions have been provided for an approach to minimize, and in particular, assess SEI and its deleterious effect on accuracy and sensitivity, and hence address the validity of quantitative results. Consideration is given to a strategy to test for SEI, and in particular, the number of samples from different sources that are required to adequately test for SEI is considered.

\section{METHODS}

A review of HPLC-MS/MS drug assay methods supporting therapeutic drug monitoring was conducted. From these, the papers used in this review were selected as follows:

\section{Search Strategy and Study Selection}

Studies eligible for consideration for this review were any articles reporting on drug assay methods using HPLC$\mathrm{MS} / \mathrm{MS}$ and/or matrix effects, with no limits placed on the date of publication. PubMed, Google Scholar, EMBASE, and MEDLINE databases were searched. Search terms included "ion suppression," "ion enhancement," "ionization suppression," "ionization enhancement," "bioanalytical analysis," and "matrix effects," with relation to tandem mass spectrometry (HPLC-MS/MS).

\section{Inclusion and Exclusion Criteria}

Only human biological matrices were included; studies investigating water and waste treatment, plant material, or animal studies were excluded, unless seen as demonstrating a relevant point for TDM assay development/validation not covered by the TDM literature.

Searches were current as of July 2016. Of these, a representative sample of studies was identified as covering all major aspects of SEI in a variety of biological matrices of human origin, including whole blood, plasma, serum, urine, saliva, cerebrospinal fluid, placental perfusate, brain tissue, liver samples, and tissue extracts (see Table, Supplemental Digital Content 1, http://links.lww.com/TDM/A225). Based on the references in the Supplementary Table, about half of the methods used to evaluate SEI included postcolumn infusion and postextraction addition, and about one quarter compared the response in pure solution with that in matrix. Several approaches were described to negate or minimize SEI, including modified sample extraction, modified mobile phase or sample dilution, and other instrument modification. About one quarter of the studies made use of a stable isotope internal standard, which can be used to normalize SEI. Of concern was that about one third of studies did not report any investigation of potential SEI.

\section{ION SOURCES}

Several different types of ion sources are currently in use, but electrospray ionization (ESI) is the most widely used. Atmospheric pressure chemical ionization (APCI) is the next most widely used ion source and reported to suffer less from SEI than does ESI, although not entirely without susceptibility. ${ }^{3,8}$ To understand SEI, an understanding of the primary ion sources used is necessary. A change in the relative quantitative output of ions compared with calibrators in the ion source in HPLC-MS/MS is considered as SEI.

A review of suppression of ionization in mass spectrometry by Annesley ${ }^{9}$ emphasized the need for evaluation of suppression of ionization for every mass spectrometric analysis at analyte concentrations expected for the purpose to which the assay is to be used. A series of experiments conducted by King et $\mathrm{al}^{3}$ suggest that liquid-phase processes are more likely involved in SEI than are gas-phase processes. Therefore, the physical and chemical properties of the analyte and the matrix affect the ionization efficiency and hence SEI.

\section{Electrospray Ionization}

The ESI ion source involves 3 distinctive steps (Fig. 1): (1) production of charged droplets at the capillary tip; (2) generation of a progeny of highly charged droplets with successive solvent evaporation and repeated charge-induced droplet explosion caused by electrostatic repulsion, and (3) 


\section{HPLC eluent}

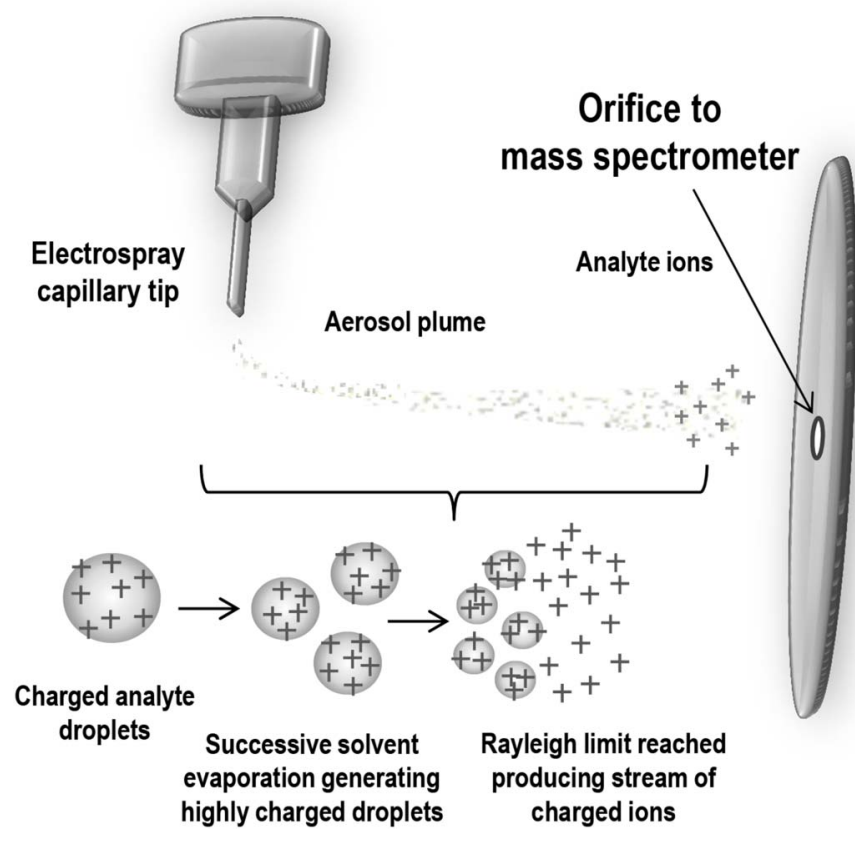

FIGURE 1. Schematic representation of ion production in electrospray ionization.

ultimate solvent evaporation, causing the electrical charges on the droplet surface to exceed the Rayleigh limit (the maximum amount of charge that a liquid droplet can carry), ${ }^{10}$ resulting in the electrostatic repulsive forces creating free charged ions in the atmosphere that are able to be drawn into the MS/MS through the orifice under the influence of an electrical field and a pressure gradient. This results in the total number of ions being limited by a concentration of around $10^{-5} \mathrm{M}$.

\section{Atmospheric Pressure Chemical Ionization}

APCI is an ionization technique using gas-phase ionmolecule reactions at atmospheric pressure, where primary ions are normally produced in a corona discharge formed around a charged needle. ${ }^{11}$ APCI differs from ESI in that the charged molecules/droplets produced at the capillary are carried into the highly chemically reactive region of the corona discharge, where the ions are formed in the gas phase, after desolvation. This method of ionization is able to handle higher HPLC eluent flow rates than ESI. Also, unlike ESI, production of ions is not restricted by the Rayleigh limit because ions form in the gas phase of the corona discharge. It does, however, compromise sensitivity for selected analytes. ${ }^{12,13}$

As described by Mallet et al, ${ }^{14}$ most applications use the ESI interface compared with APCI, with several reasons presented explaining this trend, including that ESI is an interface that is relatively easy to use, exhibits low solvent consumption, has a wide polarity range, can be applied to thermally labile compounds, and can be used for large analytes (up to $100 \mathrm{kDa}$ ).

\section{FACTORS AFFECTING IONIZATION EFFICIENCY}

SEI has largely been studied in ESI sources, and several possibilities have been suggested for the occurrence of SEI. Within the ionization source, competition between the analyte and matrix components to acquire the charge may result in a decrease or increase in concentration of the desired charged analyte ions. Also, matrix components which alter the surface activity of the droplets will limit solvation and effectively decrease the number of ionized analyte molecules available to the mass detector. ${ }^{15,16}$ The mobile-phase composition is also known to have a significant effect on ionization efficiency. Although mobile-phase additives such as formic acid may improve the sensitivity (ie, ionization efficiency) of the method, the extent of improvement depends on the nature of the additive, its concentration, and whether chromatographic separation occurs. ${ }^{17}$ The presence of nonvolatile solutes also alters the spray droplet characteristics and affects the production of further droplets, evaporation, and successive ion production. These nonvolatile solutes include salts, ionpairing agents, endogenous substances in the samples, and drug metabolites. ${ }^{9}$ Another possibility suggested for the cause of SEI is related to the individual mass and polarity of the analyte, where higher mass molecules tend to suppress the response of smaller molecules, ${ }^{18}$ and polar analytes are more liable to SEI. ${ }^{19}$ This also suggests the possibility of mutual SEI of multiple coeluting analytes in the same sample. Matrices including serum/plasma (most widely used in HPLC-MS/ MS analysis) and urine contain dissolved proteins, amino acids, and phospholipids that may also affect the ionization efficiency of the analyte. ${ }^{20}$ Phospholipids can alter ionization efficiency in both positive and negative ESI. Suppression of ionization is markedly worse when the analyte coelutes with phospholipids, most of which are late-eluting in reverse-phase methods. Despite the increased concentration of later eluting phospholipids, in reversed-phase chromatography, the early eluting lysophospholipids are more likely to cause matrix effects. $^{21}$ Given the higher number and concentration of unwanted compounds nearer the solvent front, it is to be expected that SEI is greater for analytes eluting in this region. A study to investigate matrix effects from various endogenous lipids in biological samples using common sample preparation techniques reported that none of the extraction procedures were efficient in removing all the various lipid components. $^{22}$

Although SEI affects both principle modes of ionization used in mass spectrometry, namely ESI and APCI, ESI is more susceptible. ${ }^{3,8}$ The inherent mechanisms involved in ion production in both ionization techniques contribute to their susceptibility to SEI. ${ }^{23}$ In ESI, ionization occurs in the liquid phase of the analyte, segregating the charges on the surface of the liquid droplets. As discussed above, the saturation of ionization is dependent on the available surface area of the droplets, and ionic concentrations beyond $10^{-5}$ M (Rayleigh limit) are unresponsive and sensitivity plateaus eventually decreases in response. ${ }^{24}$ In the presence of interferences, coeluting compounds and their liquid-phase properties (including concentration) may override the surface activities of the analyte of interest and reach the optimal 
ionic strength of $10^{-5} \mathrm{M}$ and subsequent signal suppression of the analyte. ${ }^{2}$

In APCI, ionization occurs in the gaseous phase, and analyte ions are generated by the corona discharge allowing for ionization suppression to be apparent only at much higher concentrations of matrix components (or coeluting substances) compared with ESI. ${ }^{24}$ However, APCI is not completely immune from the effects of SEI because the charge transfer at the corona discharge needle is dependent on the analyte/ solvent composition. ${ }^{25}$

King et $\mathrm{al}^{3}$ reported minimal SEI with APCI, compared with ESI, in a postcolumn infusion experiment. Under otherwise similar extraction processes and chromatographic conditions, Matuszewski et $\mathrm{al}^{23}$ investigated new drug candidates and found that the relative matrix effect (SEI) for compounds was small when APCI was used and very significant (up to $92 \%$ ionization suppression) when the ESI interface was used. The transformation into the gas phase is mediated by heating the gas stream in APCI, and saturation of charged ions is less likely, given the excess formation of ions. ${ }^{24}$ However, APCI encounters ionization suppression when charge transfer from the corona discharge needle is restricted by precipitation of solid material on it. In this way, the composition of the sample extract can influence the efficiency of charge transfer from the corona discharge needle. ${ }^{3}$

\section{ENHANCEMENT OF IONIZATION}

Enhancement of ionization in mass spectrometry originates from several factors that affect the ionization yield of the analyte(s) of interest, including sample constituents, and mobile-phase composition. Mobile-phase additives primarily contribute to the chromatographic resolution and also serve as ion-pairing agents. ESI signal intensity is compounddependent. Mallet et $\mathrm{al}^{14}$ conducted mass spectral analysis of terfenadine using an ESI interface with 2 different mobile-phase additives, namely ammonium hydroxide $\left(\mathrm{NH}_{4} \mathrm{OH}\right)$ and trifluoroacetic acid (TFA), and reported $75 \%$ increase in signal intensity and $75 \%$ decreased signal, respectively, compared with mass spectra of the analyte without any additives. Another enhancement of ionization study by Liang et $\mathrm{al}^{26}$ reported $9 \mathrm{drug} / \mathrm{stable}$ isotopically labeled (SIL) pairs suppressed each other's ionization responses in ESI, and this mutual effect was dependent on the internal standard concentration. ${ }^{26}$ However, in APCI, 7 of the 9 drug/SIL isotope pairs demonstrated enhancement of ionization, suggesting it is more apparent when using APCI.

\section{ASSESSMENT OF SEI}

The 2 most common approaches to evaluate SEI effects are postextraction addition and postcolumn infusion methods. In postextraction addition, the following approach is followed: a standard addition of the drug to be analyzed is made (1) into pure solution (eg, mobile phase if the extracts are redissolved in mobile phase); (2) into blank matrix (eg, plasma) before sample preparation, after which the samples are prepared according to the proposed protocol; and (3) in a second set of blank extracts after preparation. Comparison of the responses obtained from these 3 treatments allows for determination of (1) matrix effect (3/1) (including SEI), (2) extraction recovery (2/3), and (3) process efficiency $(2 / 1) .{ }^{27,28}$ The relative matrix effect, perhaps the most important parameter, can also be determined by taking the ratio of the response of the analyte divided by the response of the internal standard for treatments 3 and 1 and determining the ratio of $3 / 1$. This parameter determines whether the internal standard is/is not normalizing for any SEI that may be occurring. The second approach, namely postcolumn infusion, originally described by King et al, ${ }^{3}$ requires the constant addition of the analyte in pure solution to the mobile phase, after the column, but before the ion source. This produces a stable (but elevated) baseline. Extracts of the matrix/ces to be tested are then injected as per usual. Perturbations in the baseline of the resulting chromatogram indicate retention times at which matrix components impact the ionization of the drug. This approach should be performed using infused concentrations of drug-producing ion counts comparable with those at the higher end of the proposed assay method, as SEI is also known to be concentration dependent. ${ }^{29}$ As a point of balance, however, analyte concentrations that are too high may saturate the source and generate deceptive results. ${ }^{9}$

Another approach to quantitating the impact of SEI on an assay has been reported in 2 recent studies with melatonin $^{30}$ and thymine. ${ }^{31}$ This involves the comparison of the slopes of standard curves prepared identically in (1) pure solution and (2) matrix typical of the material to be assayed. Comparison of the slopes using external standardization indicates the extent of SEI occurring in the analyte and comparison of the responses of the internal standard indicate the extent of SEI occurring in the internal standard. Comparison of the slopes of the calibration curves using internal standardization indicates the impact of SEI on the actual assay (assuming the assay includes an internal standard).

\section{STRATEGIES TO NEGATE OR MINIMIZE IONI- ZATION SUPPRESSION AND/ OR ENHANCEMENT}

Because the mobile-phase composition is relatively constant, the impact of SEI because of sample composition is of primary importance, making effective sample extraction, preserving the analyte concentration, and optimal chromatographic conditions of significant benefit. Strategies used to negate or minimize SEI are outlined below.

\section{Modified Sample Extraction}

The degree of SEI related to each of the traditional sample preparation methods has been reported. The most common methods used in biological sample preparation are solid-phase extraction (SPE), liquid-liquid extraction (LLE), and protein precipitation. Compared with SPE or LLE methods, which are more labor intensive, protein precipitation is the simplest and easiest approach but has been shown to be the most subject to SEI. ${ }^{19}$

In LLE, the differential solubility of the analyte in 2 immiscible liquids, usually at a given $\mathrm{pH}$, results in cleaner extracts, reducing the risk of coeluting matrix components 
causing SEI. Although combination of these techniques reduces matrix interferences, a compromise in analytical recovery of the analytes is often experienced.

SPE, being itself a chromatographic technique, affords the cleanest sample extracts and unsurprisingly has been shown to demonstrate the least issues with SEI. However, extensive development procedures are required for the optimization of analyte recovery and effective extraction, based on the physicochemical properties of the analyte and the sample matrix. Sample preparation is therefore more complex, time consuming, and expensive using this technique. Targeted removal of phospholipids and proteins could be achieved using a hybrid SPE-precipitation procedure. ${ }^{32}$ Strong cation exchange SPE methods are also described to minimize ionization suppression from phospholipids. ${ }^{33}$

\section{Chromatographic Separation}

As previously indicated, SEI results from coelution of matrix components with the drug of interest. Extension of the gradient duration ${ }^{34}$ and improved efficiency of the chromatographic system by use of either a more suitable column design or use of UHPLC can all reduce the impact of ionization suppression. ${ }^{35}$ Mobile-phase $\mathrm{pH}$ adjustments, ${ }^{36}$ or the use of diverter valves to reduce entry of matrix components to the ion source, can also effectively reduce SEI and generate reproducible and accurate analytical results. The development of techniques including online 2-dimensional chromatography offers improved sample cleanup and minimizes inferences in the sample matrix. ${ }^{37}$

\section{Mobile-Phase Modification/Dilution of the Sample}

A relatively simple approach to minimize SEI is to reduce the volume of the sample injected and/or dilution of the sample extracts. Accumulation of causative agents on columns, with the eventual "bleed" from the column, will be reduced by this approach; however, the need to clean the column on a regular basis may still be required. Schuhmacher et $\mathrm{al}^{13}$ confirmed a decrease in suppression of ionization by dilution of samples with mobile phase. This approach, however, is ultimately limited by instrument sensitivity. Ferrer et $\mathrm{al}^{29}$ also demonstrated that reduced concentration of analyte also reduces suppression of ionization. Intuitively, a reduction in the concentration (mass on column) of the analyte in the extract and/or the agent causing SEI will likely reduce the impact of SEI. It is best, therefore, to perform validation for SEI at the highest analyte concentration (mass on column) expected in the assay.

Although accumulation of matrix components in the analytical column could be controlled to some extent by using a guard column or by reducing the injection volume, an organic flush after analyte elution could extend the life of the analytical column. Depending on the added concentration and the extent of chromatographic separation, mobile-phase additives can improve the ionization efficiency and minimize the suppression of ionization. ${ }^{17}$ This potential for a temporal delay in SEI until the column capacity for the agent causing SEI is exceeded, and "bleed" commences should be considered during all stages of assay development and validation of methods, as well as after initiating routine use.

\section{Novel Sample Preparation Methods}

Although the above conventional sample preparation methods, namely protein precipitation, SPE, and LLE may be effective in minimizing matrix effects, these techniques can be complex and time consuming and difficult for metabolites or biomarkers that are present at very low concentration. ${ }^{38}$ In these cases, the method must be able to preconcentrate the analyte(s) while removing all other components of the matrix. Bylda et $\mathrm{al}^{38}$ provide a summary of the latest developments in sample preparation techniques to overcome common difficulties with complex biofluids. These new developments for sample preparation methods are directed toward simplification, automation, miniaturization, and specificity enhancements of the cleanup process. ${ }^{38}$

\section{STRATEGIES TO NORMALIZE FOR SUPPRESSION AND/OR ENHANCEMENT OF IONIZATION}

\section{Use of Stable Isotope Internal Standards}

Most traditional analytical methods used in bioanalysis rely on the use of an internal standard, usually an analog of the drug being quantitated. In the case of HPLC-MS/MS, however, as previously mentioned, the potential for drug and internal standard to see differential SEI suggests that the use of an analog may well simply increase errors introduced by SEI in any given sample. The use of stable isotope internal standards has several benefits. It not only normalizes for sample-to-sample recovery differences, but it also allows for sample-to-sample (and sample-to-calibrator) differences in SEI. This, however, does not compensate for sensitivity losses that may occur. This is the most widely used approach to normalize for SEI impacts on a quantitative assay. ${ }^{39}$ SILinternal standards have been proven to deliver superior analytical results for quantitative HPLC-MS/MS assays compared with other internal standards. ${ }^{40}$ Availability and cost concerns critically limit the extent to which SIL-internal standards are used in bioanalytical methods. ${ }^{13}$

Although the use of SIL-internal standards has solved many of the issues with SEI, reports since 2003, such as the study by Jemal et al, ${ }^{41}$ have indicated circumstances in which the use of a stable isotope is not sufficient to guarantee correcting for SEI using signal normalization. In 2006, Wang et $\mathrm{al}^{42}$ highlighted this issue in an HPLC-MS/MS assay for the enantiomers of carvedilol. In this article, they described variation in the slopes of calibration curves prepared in different sources of matrix, an indicator of SEI. This occurred despite using a stable isotope internal standard. On investigation using postcolumn infusion, they identified a minor difference in retention times between the analyte and its stable isotope for one of the enantiomers. The retention time of this enantiomer was shown by postcolumn infusion to fall where the concentration of an unidentified agent causing suppression of ionization from the matrix was rapidly changing. This small reduction in retention time (approx. 1.2 seconds), known as the deuterium (or isotopic) effect, was sufficient 
TABLE 1. Differences in Physicochemical Properties of Water When Containing Deuterium

\begin{tabular}{lccc}
\hline Property & $\mathbf{D}_{\mathbf{2}} \mathbf{O}$ & HDO & $\mathbf{H}_{\mathbf{2}} \mathbf{O}$ \\
\hline Freezing point & $3.82^{\circ} \mathrm{C}$ & $2.04^{\circ} \mathrm{C}$ & $0.0^{\circ} \mathrm{C}$ \\
Boiling point & $101.4^{\circ} \mathrm{C}$ & $100.7^{\circ} \mathrm{C}$ & $100.0^{\circ} \mathrm{C}$ \\
Density at STP & 1.1056 & 1.054 & 0.9982 \\
\hline
\end{tabular}

Heavy water $\left(\mathrm{D}_{2} \mathrm{O}\right)$, semiheavy water (HDO), and light water $\left(\mathrm{H}_{2} \mathrm{O}\right)$ at standard temperature and pressure (STP).

to result in differing matrix composition in the ion source for the analyte and its stable isotope. This same phenomenon has been reported by other authors. ${ }^{40,43}$ Further investigation of the literature indicates that this phenomenon has been known since 1938 at least, ${ }^{44}$ when it was described as a means of separating stable isotopes using ion exchange chromatography. In 1999, Filer" ${ }^{45}$ reviewed "isotopic fractionation of organic compounds in chromatography" and reported over 300 references. Given the high-efficiency HPLC columns used today, the appearance of this phenomenon is not so surprising. The data in Table 1 demonstrate the differences in physicochemical properties of water when deuterium replaces hydrogen in the molecule, demonstrating the basis of the isotope effect.

The isotopes of ${ }^{13} \mathrm{C},{ }^{15} \mathrm{~N}$, and ${ }^{18} \mathrm{O}$ are reported to mimic the analyte to a greater extent compared with deuterated isotopes in chromatographic separation. A UHPLC-MS/MS $\operatorname{method}^{46}$ for determination of amphetamine and methamphetamine compared the use of ${ }^{13} \mathrm{C}$ and ${ }^{2} \mathrm{H}$ SIL to study SEI and reported better control of SEI with the use of the ${ }^{13} \mathrm{C}$-labeled isotope. Reverse-phase and hydrophilic interaction liquid chromatographic separations were studied with evidence of deuterium effect with the use of ${ }^{2} \mathrm{H}$ internal standard when reverse-phase chromatography was used. ${ }^{47}$ Table 2 highlights the percentage mass changes in common stable isotope atoms; these data are consistent with the different behavior seen between isotopes reported above. On this basis, isotopes other than deuterium may be preferable. Unfortunately, the commercial availability of isotopes other than the deuterated form is limited.

Variable extraction recoveries (between the analyte and its SIL-internal standard) and instability of SIL-internal standards can also, on rare occasions, contribute to varying results and compromised accuracy. ${ }^{48}$ The consistency of the analyte/IS ratio, imperative for a robust bioanalytical method, is therefore never satisfied with the use of SIL-internal standards. It is therefore necessary to validate for SEI, even when using stable isotope internal standards.

TABLE 2. Percent Mass Changes in Common Stable Isotope Atoms Used in Stable Isotope Internal Standards

\begin{tabular}{ll}
\hline Change in Stable Isotope & Percentage Increase in Mass \\
\hline $1 \mathrm{H}>2 \mathrm{H}$ & $100 \%$ increase in mass \\
$12 \mathrm{C}>13 \mathrm{C}$ & $1 / 12 \times 100=8 \%$ increase in mass \\
$14 \mathrm{~N}>15 \mathrm{~N}$ & $1 / 14 \times 100=7 \%$ increase in mass \\
\hline
\end{tabular}

\section{CONCLUSION}

Although we are far from a complete understanding of the chemistry involved in ion formation in the ion sources, especially in the widely used ESI source, our understanding of ion formation and the factors affecting it is growing rapidly. This, however, has had the impact of making method development and validation more complex. The increasing sensitivity of emerging tandem mass spectrometers is addressing at least one source of this problem, where the decreasing mass "on-column" reduces issues with perturbation of ionization in the ion source. It is likely that source design will also reduce the impact of perturbation of ion efficiency in the future. In the meantime, method development and validation needs to include adequate means of minimizing and assessing for SEI. Several approaches have been reviewed, including investigation using postcolumn infusion using several sample matrices, monitoring of interfering matrix components (phospholipid) transitions along the entire chromatographic run, and monitoring the stability of the internal standard when the internal standard is a SIL-internal standard.

If better understood, enhancement of ionization has the potential to be used in a beneficial manner, improving sensitivity, and further study in this area is undoubtedly warranted.

It should be noted that sources of coeluting compounds that may cause SEI may be either endogenous or exogenous. Endogenous sources can reasonably be expected to be present in all samples. If present in consistent amounts, such compounds are only likely to impact the sensitivity of a given assay with limited impact on the accuracy, when quantitated against calibrators prepared in similar matrix. If the concentration of the agent causing SEI varies significantly between matrices obtained from different subjects; however, the impact on accuracy may be substantial, resulting in erroneous results. The other sources of coeluting agents that may cause disturbances in ion efficiency are exogenous compounds in the form of concomitant medications, complementary medicines, or dietary sources. ${ }^{49}$ Given the random nature of these latter sources of interferents that may cause SEI, a rigorous consideration of the number of samples to be assessed should be made.

The development and validation of a robust, reproducible, and accurate analytical method for analysis of biological samples is always challenging, given the diversity and complexity of sample matrices. The significance of understanding and mitigating SEI has been a concern since the earliest publications. The issue of SEI has somewhat tarnished the reputation of HPLC-MS/MS but can be overcome by well-informed analysts, allowing for access to all of the other benefits of HPLC-MS/MS, including sensitivity, precision, and rapid turnaround times.

\section{REFERENCES}

1. Dooley KC. Tandem mass spectrometry in the clinical chemistry laboratory. Clin Biochem. 2003;36:471-481.

2. Antignac J, De Wasch K, Monteau F, et al. The ion suppression phenomenon in liquid chromatography-mass spectrometry and its consequences in the field of residue. Anal Chim Acta. 2005;529:129-136. 
3. King R, Bonfiglio R, Fernandez-Metzler C, et al. Mechanistic investigation of ionization suppression in electrospray ionization. $J$ Am Soc Mass Spectrom. 2000;11:942-950.

4. Taylor PJ. Matrix effects: the Achilles heel of quantitative highperformance liquid chromatography-electrospray-tandem mass spectrometry. Clin Biochem. 2005;38:328-334.

5. Mitchell TB, Dyer KR, Newcombe D, et al. Subjective and physiological responses among racemic-methadone maintenance patients in relation to relative (S)- vs. (R)-methadone exposure. Br J Clin Pharmacol. 2004;58: 609-617.

6. EMEA. European Medicines Agency. Guideline on bioanalytical method validation. 2011. Available at: http://www.ema.europa.eu/docs/en_GB/ document_library/Scientific_guideline/2011/08/WC500109686.pdf.

7. Furey A, Moriarty M, Bane V, et al. Ion suppression; a critical review on causes, evaluation, prevention and applications. Talanta. 2013;115:104-122.

8. Mortier KA, Verstraete AG, Zhang GF, et al. Enhanced method performance due to a shorter chromatographic run-time in a liquid chromatography-tandem mass spectrometry assay for paclitaxel. J Chromatogr A. 2004;1041:235-238.

9. Annesley TM. Ion suppression in mass spectrometry. Clin Chem. 2003; 49:1041-1044.

10. Rayleigh L. XX. On the equilibrium of liquid conducting masses charged with electricity. Philosophical Magazine Series 5. 1882;14:184-186.

11. Li DX, Gan L, Bronja A, et al. Gas chromatography coupled to atmospheric pressure ionization mass spectrometry (GC-API-MS): review. Anal Chim Acta. 2015;891:43-61.

12. Larger PJ, Breda M, Fraier D, et al. Ion-suppression effects in liquid chromatography-tandem mass spectrometry due to a formulation agent, a case study in drug discovery bioanalysis. J Pharm Biomed Anal. 2005; 39:206-216

13. Schuhmacher J, Zimmer D, Tesche F, et al. Matrix effects during analysis of plasma samples by electrospray and atmospheric pressure chemical ionization mass spectrometry: practical approaches to their elimination. Rapid Commun Mass Spectrom. 2003;17:1950-1957.

14. Mallet CR, Lu Z, Mazzeo JR. A study of ion suppression effects in electrospray ionization from mobile phase additives and solid-phase extracts. Rapid Commun Mass Spectrom. 2004;18:49-58.

15. Yamaguchi J, Ohmichi M, Jingu S, et al. Utility of postcolumn addition of 2-(2-methoxyethoxy)ethanol, a signal-enhancing modifier, for metabolite screening with liquid chromatography and negative ion electrospray ionization mass spectrometry. Anal Chem. 1999;71:5386-5390.

16. Stuber M, Reemtsma T. Evaluation of three calibration methods to compensate matrix effects in environmental analysis with LC-ESI-MS. Anal Bioanal Chem. 2004;378:910-916.

17. Choi BK, Hercules DM, Gusev AI. LC-MS/MS signal suppression effects in the analysis of pesticides in complex environmental matrices. Fresenius J Anal Chem. 2001;369:370-377.

18. Sterner JL, Johnston MV, Nicol GR, et al. Signal suppression in electrospray ionization Fourier transform mass spectrometry of multicomponent samples. J Mass Spectrom. 2000;35:385-391.

19. Bonfiglio R, King RC, Olah TV, et al. The effects of sample preparation methods on the variability of the electrospray ionization response for model drug compounds. Rapid Commun Mass Spectrom. 1999;13: $1175-1185$.

20. Ismaiel OA, Halquist MS, Elmamly MY, et al. Monitoring phospholipids for assessment of ion enhancement and ion suppression in ESI and APCI LC/MS/MS for chlorpheniramine in human plasma and the importance of multiple source matrix effect evaluations. J Chromatogr B Analyt Technol Biomed Life Sci. 2008;875:333-343.

21. Xia YQ, Jemal M. Phospholipids in liquid chromatography/mass spectrometry bioanalysis: comparison of three tandem mass spectrometric techniques for monitoring plasma phospholipids, the effect of mobile phase composition on phospholipids elution and the association of phospholipids with matrix effects. Rapid Commun Mass Spectrom. 2009;23: 2125-2138.

22. Ismaiel OA, Zhang T, Jenkins RG, et al. Investigation of endogenous blood plasma phospholipids, cholesterol and glycerides that contribute to matrix effects in bioanalysis by liquid chromatography/mass spectrometry. $J$ Chromatogr B Analyt Technol Biomed Life Sci. 2010;878:3303-3316.

23. Matuszewski BK, Constanzer ML, Chavez-Eng CM. Strategies for the assessment of matrix effect in quantitative bioanalytical methods based on HPLC-MS/MS. Anal Chem. 2003;75:3019-3030.
24. Bruins $\mathrm{CH}$, Jeronimus-Stratingh CM, Ensing K, et al. On-line coupling of solid-phase extraction with mass spectrometry for the analysis of biological samples. I. Determination of clenbuterol in urine. J Chromatogr A. 1999;863:115-122.

25. Gosetti F, Mazzucco E, Zampieri D, et al. Signal suppression/enhancement in high-performance liquid chromatography tandem mass spectrometry. J Chromatogr A. 2010;1217:3929-3937.

26. Liang HR, Foltz RL, Meng M, et al. Ionization enhancement in atmospheric pressure chemical ionization and suppression in electrospray ionization between target drugs and stable-isotope-labeled internal standards in quantitative liquid chromatography/tandem mass spectrometry. Rapid Commun Mass Spectrom. 2003;17:2815-2821.

27. Buhrman DL, Price PI, Rudewiczcor PJ. Quantitation of SR 27417 in human plasma using electrospray liquid chromatography-tandem mass spectrometry: a study of ion suppression. J Am Soc Mass Spectrom. 1996;7:1099-1105

28. Fu I, Woolf EJ, Matuszewski BK. Effect of the sample matrix on the determination of indinavir in human urine by HPLC with turbo ion spray tandem mass spectrometric detection. J Pharm Biomed Anal. 1998;18: 347-357.

29. Ferrer C, Lozano A, Aguera A, et al. Overcoming matrix effects using the dilution approach in multiresidue methods for fruits and vegetables. $J$ Chromatogr A. 2011;1218:7634-7639.

30. Khan SA, George R, Charles BG, et al. Monitoring salivary melatonin concentrations in children with sleep disorders using liquid chromatography-tandem mass spectrometry. Ther Drug Monit. 2013; 35:388-395.

31. Ni M, Duley J, George R, et al. Simultaneous determination of thymine and its sequential catabolites dihydrothymine and beta-ureidoisobutyrate in human plasma and urine using liquid chromatography-tandem mass spectrometry with pharmacokinetic application. J Pharm Biomed Anal. 2013;78-79:129-135.

32. Pucci V, Di Palma S, Alfieri A, et al. A novel strategy for reducing phospholipids-based matrix effect in LC-ESI-MS bioanalysis by means of HybridSPE. J Pharm Biomed Anal. 2009;50:867-871.

33. Shen JX, Motyka RJ, Roach JP, et al. Minimization of ion suppression in LC-MS/MS analysis through the application of strong cation exchange solid-phase extraction (SCX-SPE). J Pharm Biomed Anal. 2005;37:359-367.

34. Romanyshyn LA, Tiller PR. Ultra-short columns and ballistic gradients: considerations for ultra-fast chromatographic liquid chromatographic-tandem mass spectrometric analysis. J Chromatogr A. 2001;928:41-51.

35. Wren SA, Tchelitcheff P. Use of ultra-performance liquid chromatography in pharmaceutical development. J Chromatogr A. 2006;1119:140-146.

36. Weaver R, Riley RJ. Identification and reduction of ion suppression effects on pharmacokinetic parameters by polyethylene glycol 400 . Rapid Commun Mass Spectrom. 2006;20:2559-2564.

37. Pascoe R, Foley JP, Gusev AI. Reduction in matrix-related signal suppression effects in electrospray ionization mass spectrometry using on-line twodimensional liquid chromatography. Anal Chem. 2001;73:6014-6023.

38. Bylda C, Thiele R, Kobold U, et al. Recent advances in sample preparation techniques to overcome difficulties encountered during quantitative analysis of small molecules from biofluids using LC-MS/MS. Analyst. 2014;139:2265-2276.

39. Salm P, Taylor PJ, Lynch SV, et al. A rapid HPLC-mass spectrometry cyclosporin method suitable for current monitoring practices. Clin Biochem. 2005;38:667-673.

40. Stokvis E, Rosing H, Beijnen JH. Stable isotopically labeled internal standards in quantitative bioanalysis using liquid chromatography/mass spectrometry: necessity or not? Rapid Commun Mass Spectrom. 2005;19: 401-407.

41. Jemal M, Schuster A, Whigan DB. Liquid chromatography/tandem mass spectrometry methods for quantitation of mevalonic acid in human plasma and urine: method validation, demonstration of using a surrogate analyte, and demonstration of unacceptable matrix effect in spite of use of a stable isotope analog internal standard. Rapid Commun Mass Spectrom. 2003;17:1723-1734.

42. Wang S, Cyronak M, Yang E. Does a stable isotopically labeled internal standard always correct analyte response? A matrix effect study on a LC/MS/MS method for the determination of carvedilol enantiomers in human plasma. J Pharm Biomed Anal. 2007;43:701-707. 
43. Gabler J, Yuan C, Woroniecki W, et al. A sensitive and interference-free liquid chromatography tandem mass spectrometry method for measuring metanephrines in plasma. J Chromatogr Sep Tech. 2012;4. Available at: https://www.omicsonline.org/a-sensitive-and-interference-free-liquidchromatography-tandem-mass-spectrometry-method-for-measuringmetanephrines-in-plasma-2157-7064.1000195.php?aid=16517. Accessed January 2, 2018.

44. Taylor TI, Urey HC. Fractionation of the lithium and potassium isotopes by chemical exchange with zeolites. J Chem Phys. 1938;6: 429-438.

45. Filer $\mathrm{CN}$. Isotopic fractionation of organic compounds in chromatography. J Labelled Comp Radiopharm. 1999;42:169-197.
46. Berg T, Strand DH. ${ }^{13} \mathrm{C}$ labelled internal standards - a solution to minimize ion suppression effects in liquid chromatography-tandem mass spectrometry analyses of drugs in biological samples? $J$ Chromatogr A. 2011;1218:9366-9374.

47. Guo K, Ji C, Li L. Stable-isotope dimethylation labeling combined with LC-ESI MS for quantification of amine-containing metabolites in biological samples. Anal Chem. 2007;79:8631-8638.

48. Wieling J. LC-MS-MS experiences with internal standards. Chromatographia. 2002;55:S107-S113.

49. Leverence R, Avery MJ, Kavetskaia O, et al. Signal suppression/ enhancement in HPLC-ESI-MS/MS from concomitant medications. $B i$ omed Chromatogr. 2007;21:1143-1150. 